\title{
BMJ Open In utero exposure to alcohol and puberty in boys: a pregnancy cohort study
}

Linn Berger Håkonsen, ${ }^{1}$ Mette Louise Brath-Lund, ${ }^{2}$ Marie Louise Hounsgaard, ${ }^{2}$ Jørn Olsen, ${ }^{1}$ Andreas Ernst, ${ }^{1}$ Ane Marie Thulstrup, ${ }^{2}$ Bodil Hammer Bech, ${ }^{1}$ Cecilia Høst Ramlau-Hansen ${ }^{1}$

To cite: Håkonsen LB, BrathLund ML, Hounsgaard ML, et al. In utero exposure to alcohol and puberty in boys: a pregnancy cohort study. BMJ Open 2014;4:e004467. doi:10.1136/bmjopen-2013004467

\section{- Prepublication history for this paper is available online. To view these files please visit the journal online (http://dx.doi.org/10.1136/ bmjopen-2013-004467).}

Received 18 November 2013 Revised 5 May 2014 Accepted 13 May 2014

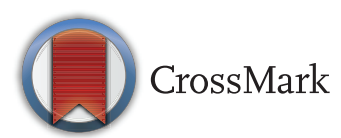

${ }^{1}$ Department of Public Health, Section for Epidemiology, Aarhus University, Aarhus C, Denmark

${ }^{2}$ Department of Occupational Medicine, Danish Ramazzini Center, Aarhus University Hospital, Aarhus C, Denmark

Correspondence to Linn Berger Håkonsen; linnhaak@rm.dk

\section{ABSTRACT}

Objectives: Epidemiological studies have raised concerns about the reproductive consequences of in utero exposure to alcohol. Maternal lifestyle factors have been associated with altered pubertal development, but the impact of prenatal alcohol exposure on male puberty is unknown. Thus, the objective was to explore whether prenatal alcohol exposure alters pubertal development in boys.

Setting: Follow-up of a Danish pregnancy cohort. Participants: Sons $(\mathrm{N}=2522)$ of women who were enrolled in a Danish pregnancy cohort between 1984 and 1987.

\section{Primary and secondary outcome measures:}

Indicators of pubertal development, assessed by age at first nocturnal emission, voice break, acne and regular shaving.

Results: We found a tendency towards a later age at first nocturnal emission and voice break following in utero exposure to binge drinking. Boys exposed to $\geq 5$ binge drinking episodes during pregnancy experienced their first nocturnal emission 7.3 months $(95 \% \mathrm{Cl}$ -2.8 to 17.4$)$ later and voice break 4.9 months $(95 \%$ $\mathrm{Cl}-0.6$ to 10.4) later than the unexposed boys. Results for average weekly alcohol consumption were in the same direction, but differences were smaller and not statistically significant.

Conclusions: We found no strong support for the hypothesis that in utero exposure to weekly alcohol consumption is a risk factor for altered pubertal development, but a tendency towards delayed pubertal development among boys exposed to binge drinking during fetal life was observed. Longitudinal studies, with data collected as children go through puberty, are needed to explore this further.

\section{INTRODUCTION}

Alcohol consumption during pregnancy has been associated with adverse pregnancy outcomes $^{1}{ }^{2}$ and child morbidity. ${ }^{3}$ Although controversy still exists regarding the safety of low-level consumption ${ }^{4}$ and specific timing of exposure, women are advised to abstain from alcohol when planning to conceive and throughout pregnancy. Yet, alcohol consumption is common during pregnancy in many

\section{Strengths and limitations of this study}

Strengths

- Large pregnancy cohort with a rather high participation rate $(55 \%)$.

- Prospectively collected data on maternal alcohol consumption.

- Data with a large exposure contrast.

- Ability to study dose-response effects.

- 'State-of-the-art' statistical methods.

Limitations

- Self-reported data on pubertal development.

- Lack of valid indicators of pubertal development in boys.

- A relatively large amount of missing data on the indicators of pubertal development.

- Risk of uncontrolled or residual confounding.

countries $^{5}{ }^{6}$ and is, thus, one of the major modifiable risk factors possibly affecting fetal growth.

Interest in male reproductive consequences of prenatal exposure to alcohol has grown recently, as focus on causes of subfecundity has intensified worldwide. In boys, one study reported an association between prenatal alcohol exposure and cryptorchidism, ${ }^{7}$ a congenital malformation that may predispose to impaired semen quality, whereas other studies have shown inconsistent results. ${ }^{8-10}$ In adult life, indications of reduced semen quality among exposed men have been reported. ${ }^{11}$

A few studies have investigated the association between maternal alcohol consumption during pregnancy and pubertal development in girls. One of these observed a later age of menarche in a small group of heavily exposed girls, ${ }^{12}$ but two recent studies did not support this finding. ${ }^{13}{ }^{14}$ Shrestha et $a l^{14}$ also assessed binge drinking episodes during pregnancy, but found no effects on the timing of menarche, either of binge drinking or of weekly alcohol consumption.

In boys, studies on risk factors for altered pubertal development are sparse. It is well 
established that variability in onset of puberty depends on genetic factors, ethnicity and nutritional conditions, ${ }^{15}$ but in utero exposures may also have an early 'programming' role. It has been indicated that male pubertal development may be accelerated following in utero exposure to cigarette smoking $^{16-18}$ and maternal obesity. $^{19}$

This study aims to explore whether prenatal alcohol exposure alters pubertal development in boys.

\section{METHODS}

This study is based on data from the pregnancy cohort 'Healthy habits for two'20 established between 1984 and 1987 in the municipalities of Aalborg and Odense, Denmark. The cohort included 11980 pregnant women ( $87 \%$ of all invited), who at a routine visit to the midwife around the 36th gestational week, completed a questionnaire concerning lifestyle, demographic and health-related characteristics before and during pregnancy, including questions on consumption of alcoholic beverages during pregnancy. These women were all Danish citizens and since only $1 \%$ of Danish women aged 20-34 years were immigrants or descendants from immigrants during 1984-1987, the participants were most likely Caucasians. ${ }^{21}$ Of the 11980 pregnant women, 11144 delivered live born singletons. Of these, 5716 were boys. In 2005, 5142 men (between 18 and 21 years of age) who were alive and living in Denmark were identified in the Danish Civil Registration System and invited to complete an internet-based questionnaire. A total of $2810(55 \%)$ men responded.

\section{Exposure assessment}

In the questionnaire completed by the pregnant women around the 36th gestational week, they were asked about their average weekly intake of beer, wine and spirits while being pregnant. One drink was defined as one bottle of beer $(0.33 \mathrm{~L})$, one glass of wine or one glass of spirits. We calculated the weekly intake of these alcoholic beverages for each woman. The average maternal alcohol intake during pregnancy was categorised into four groups: 0, 0.5-1.5, 2-4 or >4 drinks/week. Furthermore, the women were asked how many times they had consumed eight or more alcoholic drinks on a single occasion (defined as binge drinking) while being pregnant with the following predetermined response categories: 0, 1-4, 5-9, 10-19 or >20 times. We formed three groups according to the number of binge drinking episodes; 0, 1-4 and $\geq 5$ times during pregnancy.

\section{Assessment of pubertal development in boys}

In the follow-up questionnaire administered to the men in 2005, four questions concerning different indicators of pubertal development were asked: "Have you had acne?," "Has your voice broken?," "Have you started to shave regularly?" and "Have you had your first nocturnal emission?". If they answered 'yes', they were also asked to provide the age in years and months at which the event first occurred. We converted the month into a fraction of a year and added years to create a continuous outcome variable for each of the four events.

\section{Covariates}

Potential confounders were identified a priori: maternal age in years (continuous), maternal pre-pregnancy body mass index (BMI; <18.5, 18.5-24.9 and $>24.9 \mathrm{~kg} / \mathrm{m}^{2}$ ), maternal smoking during pregnancy (smoker, former smoker (stopped before pregnancy) and non-smoker), maternal chronic diseases (diabetes mellitus, epilepsy, arthrosis, heart disease, cancer, psychiatric disorders, allergy or other chronic diseases combined into one variable: present vs not present), municipality of residence at the time of delivery (urban areas vs rural areas), family socioeconomic status based on the highest ranking of job description or academic background between parents at the time of pregnancy (white-collar workers, blue-collar workers and unemployed or students) and cohabitation status of the parents at birth (mother living with the father of the child vs mother not living with the father of the child).

\section{Statistical analyses}

\section{Missing information}

The number $(\%)$ of participants who gave information on age for the four outcome variables were: acne 1804 $(64 \%)$, voice break $1696(60 \%)$, regular shaving 2128 $(76 \%)$ and first nocturnal emission 924 (33\%). About three-quarter of these only provided age in years. Information on maternal average weekly alcohol consumption was complete and there were only four $(0.1 \%)$ missing values on binge drinking. Furthermore, the level of missing values in covariates varied from $0 \%$ to $6.9 \%$ (table 1). Unexposed boys had more missing values in the pubertal events compared with boys exposed to alcohol during pregnancy. However, differences were rather small.

Since complete case analysis can lead to biased estimates and limited power, we addressed the missing data problem by using multiple imputations, which often yield unbiased and more precise estimates if data are missing at random. ${ }^{22}{ }^{23}$ Briefly, the multiple imputations model is an approach that creates several $(m>1)$ different imputed datasets based on other known paticipant characteristics from the whole dataset. The $m$ complete datasets are then analysed and the results are combined using the so-called Rubin's rule, thus producing a single set of inferences that includes the variability associated with the missing data.

Prior to multiple imputations, we excluded men who had not provided age in years for one of the four events $(n=288)$. We performed multiple imputations using an interval regression imputation model (100 imputed datasets) with interval censoring of the indicators of pubertal events. The following variables were included in the main imputation model: age at first nocturnal emission, age at 
Table 1 Maternal characteristics according to average weekly alcohol consumption and binge drinking episodes

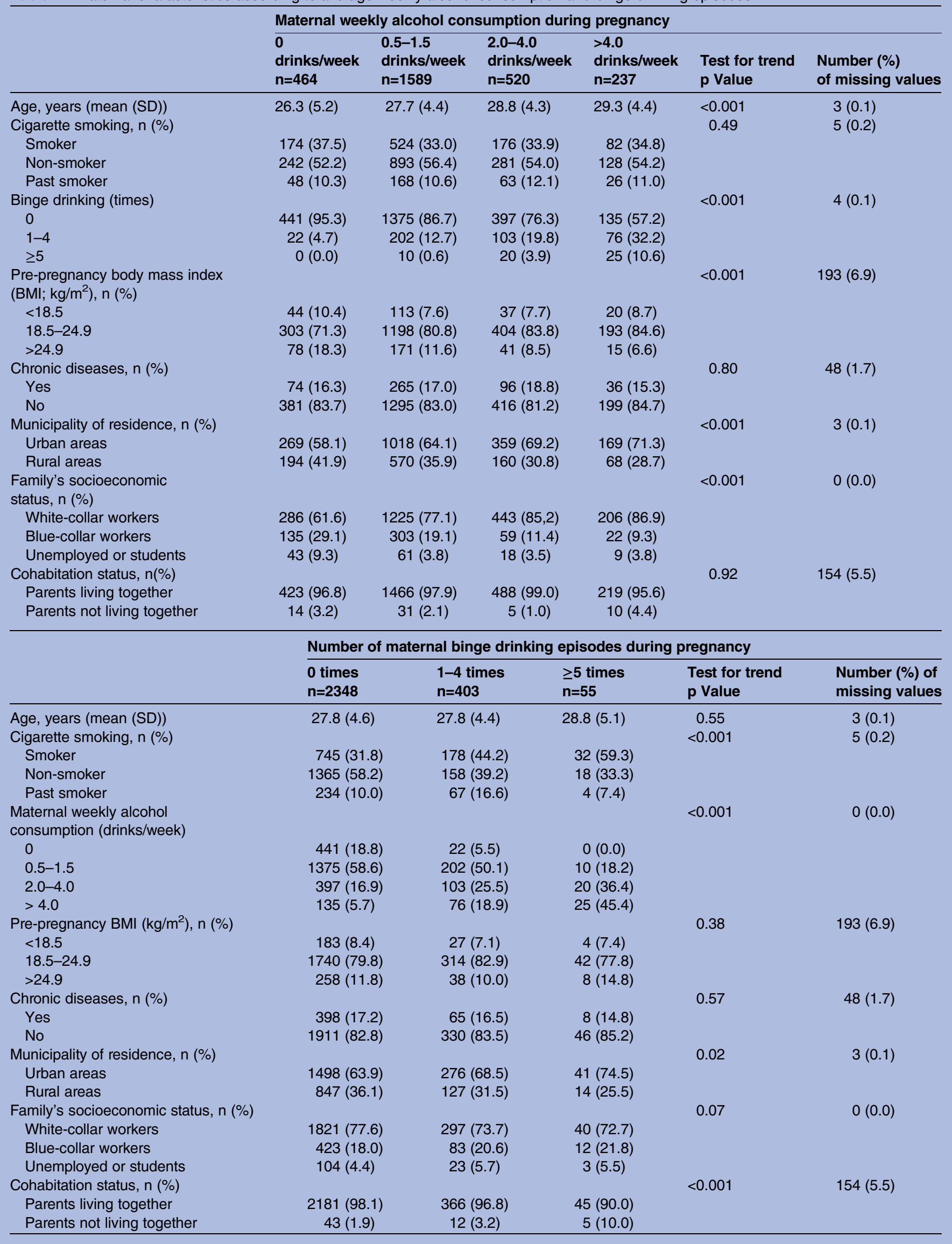


acne, age at voice break, age at regular shaving, maternal pre-pregnancy BMI, maternal cigarette smoking, maternal age at delivery, alcohol consumption during pregnancy, chronic diseases of the mother, municipality of residence at the time of delivery, family's socioeconomic status and cohabitation of the parents. We performed different imputation models, including (1) a higher number of imputed datasets, $m=120$, (2) only exposure and outcome variables and (3) more covariates than in the main imputation model. We then compared the results to check for consistency, that is, the sensitivity of the results to the choice of model used for imputations.

\section{Data analyses}

Data on age for the four indicators of pubertal development were symmetrically distributed. Thus, we calculated the mean ages with $95 \%$ confidence intervals (CIs) for each of the pubertal events. We estimated partial correlation coefficients between the four indicators of pubertal development adjusted for the covariates described above. Furthermore, we performed multiple linear regression analyses with maternal alcohol consumption during pregnancy, with average weekly intake and number of binge drinking episodes as the explanatory variables in separate models. We adjusted for the potential confounders aforementioned. Moreover, we estimated trends using average maternal alcohol consumption and binge drinking as continuous variables. Since $2.1 \%$ of the women contributed to the cohort with more than one child, we applied robust standard error (ER) in the adjusted analyses to account for clustering.

We also performed sensitivity analyses. First, we repeated the multiple linear regression analyses using different multiple imputation models to check for consistency of the chosen imputation model, as noted before. Second, we performed restricted analyses based on the participants who reported at least age in years at all events (complete case analyses). Finally, to test for interaction between maternal weekly alcohol consumption and maternal cigarette smoking, we fitted a multiple linear regression model testing for interaction among the complete cases. All statistical analyses were performed using Stata 12 software (Stata Corporation, College Station, Texas, USA).

\section{RESULTS}

The cohort included 2810 men, but 288 men provided no information on the pubertal events and the final study population constituted 2522 participants. The excluded men did not differ from the responders with regard to in utero exposure to alcohol.

Approximately $84 \%$ of the mothers consumed alcohol on a weekly basis during pregnancy and about $9 \%$ reported having $>4$ drinks of alcohol/week. In total, $16 \%$ of the mothers had experienced at least one binge drinking episode during pregnancy, and $2 \%$ had $\geq 5$ binge drinking episodes.

In table 1, the characteristics of the pregnant women by average weekly alcohol consumption and number of binge drinking episodes are presented. Women with high average weekly alcohol consumption were on average older, had more binge drinking episodes and a lower BMI than women with no alcohol intake. Furthermore, women consuming alcohol during pregnancy had higher socioeconomic status and were more likely to live in urban areas, compared with abstainers. Women binge drinking $\geq 5$ times during pregnancy smoked more often, had a higher weekly alcohol intake during pregnancy and were more likely to live in urban areas and not live with the father of the child.

The crude mean $(95 \% \mathrm{CI})$ ages for the four indicators of pubertal development among all participants were: acne 14.6 (14.5 to 14.7) years, voice break 14.5 (14.5 to 14.6) years, start of regular shaving 17.2 (17.2 to 17.3) years and first nocturnal emission 14.8 (14.7 to 14.9) years. The adjusted correlation coefficients between the four pubertal milestones varied between 0.3 and 0.6 (table 2).

We observed a tendency for a later age at all four indicators of pubertal development with higher weekly average levels of alcohol intake; however, the differences were small and not statistically significant (table 3). Compared with the unexposed boys, those exposed to $>4$ drinks/week during fetal life were 0.30 (95\% CI -0.19 to 0.80 ) years older at first nocturnal emission, corresponding to 3.6 (95\% CI -2.3 to 9.6) months. Regarding binge drinking during pregnancy, we observed a somewhat stronger indication of an older age at all four indicators of pubertal development than seen for weekly average alcohol consumption. Boys exposed to $\geq 5$ binge drinking episodes during pregnancy experienced their first

Table 2 Adjusted ${ }^{*}$ correlation between age at indicators of pubertal development

\begin{tabular}{lllll}
\hline & Acne & Voice break & Regular shaving & First nocturnal emission \\
\hline Acne & 1.00 & & & \\
Voice break & 0.60 & 1.00 & 1.00 & \\
Regular shaving & 0.30 & 0.38 & 0.30 & 1.00 \\
First nocturnal emission & 0.34 & 0.40 & & \\
\hline
\end{tabular}

${ }^{*}$ Results are adjusted for maternal alcohol consumption during pregnancy, maternal age at delivery, maternal pre-pregnancy body mass index, maternal smoking during pregnancy, maternal chronic diseases, municipality of residence at the time of delivery, family's socioeconomic status and cohabitation status of the parents at birth. 
Table 3 Age difference in years of indicators of pubertal development among 2522 boys according to average weekly alcohol intake and binge drinking episodes during pregnancy

\begin{tabular}{|c|c|c|c|c|c|}
\hline & \multirow{2}{*}{$\begin{array}{l}\text { Distribution } \\
(\%)\end{array}$} & $\begin{array}{l}\text { First nocturnal } \\
\text { emission }\end{array}$ & Acne & \multirow[t]{2}{*}{ Voice break } & \multirow[t]{2}{*}{ Regular shaving } \\
\hline & & Adjusted* mean diffe $^{*}$ & rence $(95 \% \mathrm{Cl})$ & & \\
\hline \multicolumn{6}{|c|}{ Average alcohol intake (drinks/week) } \\
\hline 0 & 16 & reference & reference & reference & reference \\
\hline $0.5-1.5$ & 56 & $0.15(-0.13$ to 0.43$)$ & $0.06(-0.12$ to 0.24$)$ & $-0.01(-0.19$ to 0.16$)$ & $0.02(-0.15$ to 0.19$)$ \\
\hline $2.0-4.0$ & 19 & $0.25(-0.12$ to 0.62$)$ & $0.09(-0.13$ to 0.31$)$ & 0.11 ( -0.10 to 0.32$)$ & $0.03(-0.18$ to 0.23$)$ \\
\hline$>4.0$ & 9 & $0.30(-0.19$ to 0.80$)$ & $0.09(-0.19$ to 0.37$)$ & $0.03(-0.24$ to 0.31$)$ & $0.08(-0.16$ to 0.33$)$ \\
\hline $\begin{array}{l}\text { Test for trend, } \\
p \text { Value }\end{array}$ & & 0.97 & 0.23 & 0.50 & 0.30 \\
\hline \multicolumn{6}{|c|}{ Binge drinking episodes (times) } \\
\hline 0 & 84 & reference & reference & reference & reference \\
\hline $1-4$ & 14 & $-0.09(-0.39$ to 0.21$)$ & $-0.02(-0.21$ to 0.17$)$ & $-0.07(-0.24$ to 0.11$)$ & $0.03(-0.14$ to 0.20$)$ \\
\hline$\geq 5$ & 2 & $0.61(-0.23$ to 1.45$)$ & $0.06(-0.42$ to 0.53$)$ & $0.41(-0.05$ to 0.87$)$ & $0.17(-0.20$ to 0.54$)$ \\
\hline $\begin{array}{l}\text { Test for trend, } \\
p \text { Value }\end{array}$ & & 0.36 & 0.95 & 0.45 & 0.37 \\
\hline
\end{tabular}

*Adjusted for maternal age at delivery, maternal pre-pregnancy BMI, maternal smoking during pregnancy, maternal chronic diseases, municipality of residence at the time of delivery, family's socio-economic and cohabitation status of the parents at birth.

nocturnal emission 7.3 (95\% CI -2.8 to 17.4 ) months later and voice break 4.9 (95\% CI -0.6 to 10.4$)$ months later than the unexposed boys. Differences between the groups for the other pubertal milestones were smaller.

We repeated all analyses based on four alternative imputation models and found essentially the same results as those presented in table 3 (data not shown). For the restricted analyses (complete cases), results were in the same direction (data not shown). Finally, in the interaction analyses, there was no indication of effect measure modification by maternal cigarette smoking (data not shown).

\section{DISCUSSION}

We found no strong evidence for an association between maternal alcohol consumption during pregnancy and pubertal development among sons, but our results indicated that binge drinking during pregnancy may be associated with pubertal development occuring at a later age in boys.

Our findings of slightly delayed pubertal development in exposed boys are consistent with results from experimental studies. Exposure to ethanol in utero has been linked to delayed sexual maturation in female rats ${ }^{24} 25$ and, recently, prenatally exposed male rats also showed delayed reproductive development and onset of spermatogenesis compared with the unexposed rats. ${ }^{26}$ Thus, it is plausible that prenatal exposure to alcohol could affect onset of spermatogenesis in humans. Evidence from epidemiological studies on pubertal development in girls is, however, inconsistent. In a small preliminary investigation by Robe $e t a l,{ }^{12}$ there were a higher percentage of girls with late onset of menarche among girls exposed to $\geq 2$ drinks of alcohol/day. Similar results were observed by Windham et $a l^{13}$ when comparing highly exposed and low exposed girls; however, after adjusting for potential confounding factors the effect on age of menarche diminished. Further, Shrestha et al ${ }^{14}$ reported no association between in utero exposure to alcohol and age of menarche. The results by Robe et al may well have been confounded, but the discrepant findings could be due to differences in exposure levels. The levels of exposure in the study by Windham et al and Shrestha et al may have been too low to detect effects.

In this present study, we used prospectively collected information from the mothers on alcohol intake during pregnancy. This limits the risk of differential recall bias; however, there is a considerable risk of non-differential recall bias driving effect measures towards the null. The prevalence of drinking in our study was high, since moderate alcohol intake during pregnancy was socially accepted in Denmark at the time of data collection. We had the ability to study dose-response effects as we had data with a large exposure contrast and, further, we had a rather large proportion (9\%) of mothers with an alcohol intake of $>4$ drinks/week. The analyses on binge drinking during pregnancy were, however, limited to a few highly exposed boys (2\%). When studying effects of prenatal alcohol exposure, one major challenge is to disentangle the toxic effects of alcohol from the underlying and possibly confounding factors associated with alcohol consumption. ${ }^{27}$ It is well established that lifetime abstainers differ from drinkers in a number of demographic, lifestyle and socioeconomic characteristics. ${ }^{28}$ Although these differences might not be expected to be as comprehensive in a study population of pregnant women, the abstainers did differ from the drinkers in our data. Although we controlled for various potential confounders, we cannot exclude residual or unmeasured confounding as an explanation for our findings.

Although the participation rate in the birth cohort was high $(87 \%)$, there is a risk of selection bias related to attrition in this study, as only $55 \%$ of the men 
participated in the follow-up in 2005. Another limitation is the relatively large amount of missing data in the cohort, especially concerning the four indicators of pubertal development. We addressed this by using multiple imputation models which provide more valid results than complete case analysis if data are missing at random. ${ }^{23}$ Also, we compared estimates from different imputation models.

The data on indicators of pubertal development were based on self-reports at the age of 18-21 years. One may argue that acne or the need for regular shaving may not occur in all men and these two events may therefore not be good predictors of pubertal development. On the other hand, age at first nocturnal emission and voice break are considered valid indicators of pubertal development in boys, comparably to age of menarche in girls. ${ }^{29} 30$ Previous data from Danish boys have shown that the median age at first ejaculation (spermache) was 13.4 years, ${ }^{31}$ and mean age at voice break was 14 years, ${ }^{32}$ which is slightly earlier than in the present study. This small discrepancy may well be explained by the recall time in our study which varied between 1 and 12 years. We expect some misclassification, yet most likely nondifferential, resulting in bias in the null direction.

Despite the high prevalence of alcohol consumption reported in this study, the number of boys exposed to binge drinking was low, and the results must therefore be interpreted with caution. There may well be a threshold for both dose and timing of exposure that may affect male pubertal development. It is plausible that the detrimental effect varies by gestational age and there may be more than one vulnerable time window.

In this study population, there was not sufficient information to study very high levels of exposure to alcohol during fetal life. Furthermore, we only measured the average number of drinks per week or number of binge drinking episodes during the entire pregnancy and did not have data on the exact time of alcohol consumption. Therefore, we cannot distinguish between early or late exposure in pregnancy and this may mask potential effects of prenatal exposure to high levels of alcohol in vulnerable time windows.

It is biologically plausible that exposure to alcohol in fetal life delays pubertal development. Differentiation and development of the male genitals begins around gestational weeks 7-8, and evidence from experimental studies suggests a window for 'male programming' occurring from weeks 8-14 of gestation, where sufficient androgen levels are essential for normal development. ${ }^{33-37}$ Alcohol readily passes the placental barrier, thereby possibly affecting the endocrine organs of the developing fetus. ${ }^{39}$ Alcohol intake during pregnancy has been shown to increase oestrogen levels and decrease testosterone levels in maternal and umbilical blood, ${ }^{39-41}$ thus, the intrauterine hormonal milieu and consequently, the fetal hormone balance may well be affected. ${ }^{38} 42$ Although the exact mechanisms are not well understood, the onset of puberty in boys is under the control of the hypothalamic-pituitary-gonadal axis $^{43}$ and alterations of the fetal hormonal milieu and endocrine system may affect pubertal development later in life. ${ }^{44}$

Further research is needed to investigate the association between maternal alcohol consumption and pubertal development. Future studies will benefit from data collected on pubertal development by following children during the years of puberty with clinical examinations, Tanner staging and assessment of changes in hormonal levels.

In summary, we found little evidence to support the hypothesis that in utero exposure to weekly alcohol consumption is a strong risk factor for altered pubertal development, but we observed a tendency towards delayed pubertal development among boys exposed to maternal binge drinking during fetal life.

Contributors LBH contributed to the design of the study, carried out the data analyses, contributed to data interpretation, drafted the manuscript and approved the final manuscript as submitted. MLBL contributed to data interpretation and drafting of the manuscript, critically reviewed the manuscript and approved the final manuscript as submitted. MLH contributed to data analyses and interpretation, critically reviewed the manuscript and approved the final manuscript as submitted. JO and $\mathrm{CHRH}$ contributed to the design of the study, acquisition of the data, data analyses and data interpretation, critically reviewed the manuscript and approved the final manuscript as submitted. AE and AMT contributed to the data interpretation, critically reviewed the manuscript and approved the final manuscript as submitted. BHB contributed to acquisition of the data, data interpretation, critically reviewed the manuscript and approved the final manuscript as submitted.

Ethics approval The study was approved by the Danish Data Protection Agency (J.nr: 2011-41-6435).

Funding A grant from the Danish Medical Research Council funded the study (271-05-0115)

Competing interests None.

Provenance and peer review Not commissioned; externally peer reviewed.

Data sharing statement No additional data are available.

Open Access This is an Open Access article distributed in accordance with the Creative Commons Attribution Non Commercial (CC BY-NC 3.0) license, which permits others to distribute, remix, adapt, build upon this work noncommercially, and license their derivative works on different terms, provided the original work is properly cited and the use is non-commercial. See: http:// creativecommons.org/licenses/by-nc/3.0/

\section{REFERENCES}

1. O'Leary CM, Nassar N, Kurinczuk JJ, et al. The effect of maternal alcohol consumption on fetal growth and preterm birth. BJOG 2009;116:390-400.

2. Nykjaer C, Alwan NA, Greenwood DC, et al. Maternal alcohol intake prior to and during pregnancy and risk of adverse birth outcomes: evidence from a British cohort. J Epidemiol Community Health 2014;68:542-9.

3. Spohr HL, Willms J, Steinhausen HC. Prenatal alcohol exposure and long-term developmental consequences. Lancet 1993;341:907-10.

4. Patra J, Bakker $\mathrm{R}$, Irving $\mathrm{H}$, et al. Dose-response relationship between alcohol consumption before and during pregnancy and the risks of low birthweight, preterm birth and small for gestational age (SGA) - a systematic review and meta-analyses. BJOG 2011;118: 1411-21.

5. Alvik A, Heyerdahl S, Haldorsen T, et al. Alcohol use before and during pregnancy: a population-based study. Acta Obstet Gynecol Scand 2006;85:1292-8. 
6. Strandberg-Larsen K, Rod NN, Nybo Andersen AM, et al. Characteristics of women who binge drink before and after they become aware of their pregnancy. Eur J Epidemiol 2008;23:565-72.

7. Damgaard IN, Jensen TK, Petersen JH, et al. Cryptorchidism and maternal alcohol consumption during pregnancy. Environ Health Perspect 2007:115:272-7.

8. Jensen MS, Bonde JP, Olsen J. Prenatal alcohol exposure and cryptorchidism. Acta Paediatr 2007;96:1681-5.

9. Mongraw-Chaffin ML, Cohn BA, Cohen RD, et al. Maternal smoking alcohol consumption, and caffeine consumption during pregnancy in relation to a son's risk of persistent cryptorchidism: a prospective study in the Child Health and Development Studies cohort, 19591967. Am J Epidemiol 2008;167:257-61.

10. Strandberg-Larsen $\mathrm{K}$, Jensen MS, Ramlau-Hansen $\mathrm{CH}$, et al. Alcohol binge drinking during pregnancy and cryptorchidism. Hum Reprod 2009;24:3211-19.

11. Ramlau-Hansen $\mathrm{CH}$, Toft $\mathrm{G}$, Jensen MS, et al. Maternal alcohol consumption during pregnancy and semen quality in the male offspring: two decades of follow-up. Hum Reprod 2010;25:2340-5

12. Robe LB, Robe RS, Wilson PA. Maternal heavy drinking related to delayed onset of daughters menstruation. Curr Alcohol 1979;7:515-20.

13. Windham GC, Bottomley C, Birner C, et al. Age at menarche in relation to maternal use of tobacco, alcohol, coffee, and tea during pregnancy. Am J Epidemiol 2004;159:862-71.

14. Shrestha A, Nohr EA, Bech BH, et al. Smoking and alcohol use during pregnancy and age of menarche in daughters. Hum Reprod 2011;26:259-65.

15. Parent AS, Teilmann G, Juul A, et al. The timing of normal puberty and the age limits of sexual precocity: variations around the world, secular trends, and changes after migration. Endocr Rev 2003;24:668-93.

16. Fried PA, James DS, Watkinson B. Growth and pubertal milestones during adolescence in offspring prenatally exposed to cigarettes and marihuana. Neurotoxicol Teratol 2001;23:431-6.

17. Ravnborg TL, Jensen TK, Andersson AM, et al. Prenatal and adult exposures to smoking are associated with adverse effects on reproductive hormones, semen quality, final height and body mass index. Hum Reprod 2011;26:1000-11.

18. Håkonsen LB, Olsen $\mathrm{J}$, Stovring $\mathrm{H}$, et al. Maternal cigarette smoking during pregnancy and pubertal development in sons. A follow-up study of a birth cohort. Andrology 2013;1:348-55.

19. Hounsgaard ML, Hakonsen LB, Vested A, et al. Maternal pre-pregnancy body mass index and pubertal development among sons. Andrology 2014;2:198-204.

20. Olsen J, Frische G, Poulsen AO, et al. Changing smoking, drinking, and eating behaviour among pregnant women in Denmark Evaluation of a health campaign in a local region. Scand J Soc Med 1989;17:277-80.

21. Statistics Denmark. FOLK2: population by sex, age, ancestry, country of origin and citizenship (1980-2013) 2013. http://www.dst.dk/en

22. Greenland S, Finkle WD. A critical look at methods for handling missing covariates in epidemiologic regression analyses. $A m \mathrm{~J}$ Epidemiol 1995;142:1255-64.

23. Sterne JA, White IR, Carlin JB, et al. Multiple imputation for missing data in epidemiological and clinical research: potential and pitfalls. BMJ 2009;338:b2393.

24. Boggan WO, Randall CL, Dodds HM. Delayed sexual maturation in female C57BL/6J mice prenatally exposed to alcohol. Res Commun Chem Pathol Pharmacol 1979;23:117-25.
25. McGivern RF, Raum WJ, Handa RJ, et al. Comparison of two weeks versus one week of prenatal ethanol exposure in the rat on gonadal organ weights, sperm count, and onset of puberty. Neurotoxicol Teratol 1992;14:351-8.

26. Lan N, Vogl AW, Weinberg J. Prenatal ethanol exposure delays the onset of spermatogenesis in the rat. Alcohol Clin Exp Res 2013;37:1074-81.

27. Strandberg-Larsen K, Andersen AM. Alcohol and fetal risk: a property of the drink or the drinker? Acta Obstet Gynecol Scand 2011;90:207-9.

28. Naimi TS, Brown DW, Brewer RD, et al. Cardiovascular risk factors and confounders among nondrinking and moderate-drinking U.S. adults. Am J Prev Med 2005;28:369-73.

29. Laron Z, Arad J, Gurewitz R, et al. Age at first conscious ejaculation: a milestone in male puberty. Helv Paediatr Acta 1980;35:13-20.

30. Carlier JG, Steeno OP. Oigarche: the age at first ejaculation. Andrologia 1985;17:104-6.

31. Nielsen CT, Skakkebaek NE, Richardson DW, et al. Onset of the release of spermatozoa (spermarche) in boys in relation to age, testicular growth, pubic hair, and height. J Clin Endocrinol Metab 1986;62:532-5.

32. Juul A, Magnusdottir S, Scheike T, et al. Age at voice break in Danish boys: effects of pre-pubertal body mass index and secular trend. Int J Androl 2007;30:537-42.

33. Hutson JM. Testicular feminization: a model for testicular descent in mice and men. J Pediatr Surg 1986;21:195-8

34. Ahmed SF, Cheng A, Dovey L, et al. Phenotypic features, androgen receptor binding, and mutational analysis in 278 clinical cases reported as androgen insensitivity syndrome. J Clin Endocrinol Metab 2000;85:658-65.

35. Welsh M, Saunders PT, Fisken M, et al. Identification in rats of a programming window for reproductive tract masculinization, disruption of which leads to hypospadias and cryptorchidism. J Clin Invest 2008;118:1479-90.

36. MacLeod DJ, Sharpe RM, Welsh M, et al. Androgen action in the masculinization programming window and development of male reproductive organs. Int J Androl 2010;33:279-87.

37. Welsh M, MacLeod DJ, Walker M, et al. Critical androgen-sensitive periods of rat penis and clitoris development. Int J Androl 2010;33: e144-52.

38. Weinberg J. Recent studies on the effects of fetal alcohol exposure on the endocrine and immune systems. Alcohol Alcohol Suppl 1994;2:401-9.

39. Petridou E, Katsouyanni K, Spanos E, et al. Pregnancy estrogens in relation to coffee and alcohol intake. Ann Epidemiol 1992;2:241-7.

40. Wuu J, Hellerstein S, Lipworth L, et al. Correlates of pregnancy oestrogen, progesterone and sex hormone-binding globulin in the USA and China. Eur J Cancer Prev 2002;11:283-93.

41. Nagata $\mathrm{C}$, Iwasa S, Shiraki M, et al. Association of maternal fat and alcohol intake with maternal and umbilical hormone levels and birth weight. Cancer Sci 2007;98:869-73.

42. Udani M, Parker S, Gavaler J, et al. Effects of in utero exposure to alcohol upon male rats. Alcohol Clin Exp Res 1985;9 355-9.

43. Lewis K, Lee PA. Endocrinology of male puberty. Curr Opin Endocrinol Diabetes Obes 2009;16:5-9.

44. Teilmann G, Juul A, Skakkebaek NE, et al. Putative effects of endocrine disrupters on pubertal development in the human. Best Pract Res Clin Endocrinol Metab 2002:16:105-21. 